\title{
Imaging of the Thyroid and Parathyroid Using a Cardiac Cadmium-Zinc-Telluride Camera: Phantom Studies
}

\author{
Yosuke Miyazaki ${ }^{1}$, Yasuhiro Kato ${ }^{1},{\text { Akira } \text { Imoto }^{2} \text {, and Kazuki Fukuchi }}^{1}$ \\ ${ }^{1}$ Division of Medical Technology and Science, Department of Medical Physics and Engineering, Course of Health Science, Osaka \\ University Graduate School of Medicine, Suita, Osaka, Japan; and ${ }^{2}$ Department of Radiology, National Cerebral and Cardiovascular \\ Center, Suita, Osaka, Japan
}

\begin{abstract}
Cadmium-zinc-telluride (CZT) detectors have recently been introduced to the field of clinical nuclear cardiology. However, the feasibility of using them for organs other than the heart remains unclear. The aim of this study was to evaluate the potential of a cardiac CZT camera to acquire thyroid and parathyroid images. We used custom-made phantoms and the currently available standard protocols for a CZT camera, instead of a sodium-iodine scintillation (Nal) camera. Methods: Thyroid phantoms with or without parathyroid adenomas were made from agar using radiopharmaceuticals $\left({ }^{99 \mathrm{~m} T c}\right.$ or $\left.{ }^{123} \mathrm{l}\right)$ and imaged using CZT and Nal cameras. Using the CZT camera data, we prepared maximumintensity-projection (MIP) images and planar-equivalent images. Image counts were compared with those from the Nal camera, and the radioactivity of the phantoms was measured. For parathyroid imaging, 3 different protocols with the Nal camera were tested using MIP images. Results: For thyroid imaging, MIP could provide images as clear as those obtained from the Nal camera. The radioactivity and image counts correlated better for the planar-equivalent images than for the MIP images, especially for ${ }^{123}$. We succeeded in obtaining clear parathyroid adenoma images from MIP images using all 3 protocols. Conclusion: A cardiac CZT camera can effectively perform qualitative and quantitative assessments of the thyroid and parathyroid organs.
\end{abstract}

Key Words: cadmium zinc telluride (CZT); thyroid imaging; parathyroid imaging; dual-isotope acquisition

J Nucl Med Technol 2018; 46:39-44

DOI: $10.2967 /$ jnmt.117.199042

$\mathbf{R}$ ecently, semiconductor cadmium-zinc-telluride (CZT) detectors have been introduced to the field of cardiovascular nuclear medicine. These CZT-based cameras have better sensitivity and spatial resolution than a conventional $\gamma$-camera composed of sodium-iodine scintillation detectors (NaI camera) (1-6). Gambhir et al. reported that a CZT camera has 10 -fold better sensitivity and approximately

Received Jul. 10, 2017; revision accepted Sep. 23, 2017.

For correspondence or reprints contact: Kazuki Fukuchi, Division of Medical Technology and Science, Department of Medical Physics and Engineering, Course of Health Science, Osaka University Graduate School of Medicine, 1-7 Yamadaoka, Suita, Osaka 565-0871, Japan.

E-mail: kfukuchi@sahs.med.osaka-u.ac.jp

Published online Nov. 10, 2017.

COPYRIGHT (c) 2018 by the Society of Nuclear Medicine and Molecular Imaging. 2-fold better spatial resolution than a NaI camera (6). This marked increase in sensitivity results in a reduction of acquisition time and radiopharmaceutical dose (1-3,5-7).

In addition, a CZT camera has better energy resolution than a NaI camera. Takahashi et al. reported that the energy resolution of a CZT camera is 5\% whereas that of a $\mathrm{NaI}$ camera is $10 \%$ (4). Therefore, CZT SPECT has the potential to separate the primary photons of ${ }^{99 \mathrm{~m}} \mathrm{Tc}$ and ${ }^{123} \mathrm{I}$, which are very close, and provides better-quality dual-tracer simultaneous imaging $(8,9)$.

Although CZT SPECT has the remarkable abovementioned advantage for nuclear medicine imaging, it is currently applied clinically only for cardiovascular and breast imaging (2). If cardiac CZT SPECT were to be used for focused imaging of other organs, the same advantages are to be expected. To make this possible, however, there are some problems that need to be overcome. First, the current cardiac CZT camera can acquire only tomographic images and not planar images. Second, the field of view of the apparatus is limited $(2,3)$. Even with these limitations, it should be possible to image small and superficial organs using a cardiac CZT camera; therefore, we assumed that the thyroid and parathyroid organs would be suitable for this imaging modality.

The aim of this study was to verify the ability to use cardiac CZT SPECT for imaging of the thyroid and parathyroid. Thyroid and parathyroid adenoma phantoms were fabricated to use in simulation studies, which were performed with current clinical thyroid and parathyroid scintigraphy protocols (10-18).

\section{MATERIALS AND METHODS}

\section{Thyroid and Parathyroid Adenoma Phantoms}

A thyroid phantom mold was made from potassium alginate and sodium alginate for modeling the normal-sized thyroid glands as a reference (19-21). The length, width, and depth of the thyroid phantom were 60,20 , and $15 \mathrm{~mm}$, respectively. Agar solution and radiopharmaceuticals were then poured into the mold and stirred until the concentration of radioactivity was homogeneous. After $30 \mathrm{~min}$, the agar solidified and was usable as a thyroid phantom. A parathyroid adenoma phantom was made using a similar method. We gave it a diameter of $8 \mathrm{~mm}$ and a cylindric height of $7 \mathrm{~mm}$ based on the size of parathyroid adenomas resected from patients $(22,23)$. This phantom was fixed to the lower quadrant of the left 
TABLE 1

Tracer Dose of Each Phantom in 3 Parathyroid Imaging Protocols

\begin{tabular}{|c|c|c|c|}
\hline Protocol & Thyroid (MBq) & Parathyroid (MBq) & Background (MBq/mL) \\
\hline \multicolumn{4}{|l|}{ Dual-phase $99 \mathrm{mTc}$-sestamibi } \\
\hline Early phase (99mTc) & 4.0 & 0.1 & 0.02 \\
\hline Delayed phase $\left({ }^{99 \mathrm{~m} T c}\right)$ & 0.5 & 0.07 & 0.014 \\
\hline \multicolumn{4}{|c|}{ Dual-tracer ${ }^{99 \mathrm{mT}} \mathrm{T}$-sestamibi/99mTc-pertechnetate } \\
\hline Single tracer $\left({ }^{99 \mathrm{~m} T c}\right)$ & 4.0 & - & 0.02 \\
\hline Dual tracer (99mTc) & 8.0 & 0.1 & 0.04 \\
\hline \multicolumn{4}{|c|}{ Dual-tracer ${ }^{99 m} T c-s e s t a m i b i /{ }^{123} \mid$-iodine } \\
\hline $99 \mathrm{mTc}$ & 4.0 & 0.1 & 0.02 \\
\hline 123 & 0.74 & - & 0.006 \\
\hline
\end{tabular}

lobe of the thyroid phantom using a needle. Thyroid phantoms with or without a parathyroid adenoma phantom were fixed in the center of a 1,500-mL cylindric plastic bottle $(30-\mathrm{cm}$ circumference) filled with tracer-containing water, which was used to simulate an adult neck with background activity.

\section{Thyroid Imaging}

We prepared several thyroid phantoms with various concentrations of ${ }^{99 \mathrm{~m}} \mathrm{Tc}$-pertechnetate or ${ }^{123} \mathrm{I}-15$-( $p$-iodophenyl)-3methylpentadecanoic acid ( ${ }^{123} \mathrm{I}$-BMIPP). In Japan, $\mathrm{Na}^{123} \mathrm{I}$ is available only in the form of a powder contained in a capsule, which is not usable for a phantom study. Therefore, we used ${ }^{123}$ I-BMIPP, a liquid radiopharmaceutical, to mix homogeneously with agar. In the thyroid phantoms, $1.85-27.8 \mathrm{MBq}$ of ${ }^{99 \mathrm{~m}} \mathrm{Tc}$-pertechnetate or $0.37-3.7 \mathrm{MBq}$ of ${ }^{123} \mathrm{I}-\mathrm{BMIPP}$ were included. Plastic bottles were filled with a $0.03 \mathrm{MBq} / \mathrm{mL}$ solution of $99 \mathrm{~m} \mathrm{Tc}$-pertechnetate or a $0.006 \mathrm{MBq} / \mathrm{mL}$ solution of ${ }^{123}$ I-BMIPP for background. These background tracer concentrations were determined using a previous thyroid phantom experiment as a reference (24). The neck phantom was placed supine, and imaging was performed first with the CZT camera and then with the NaI camera for $10 \mathrm{~min}$ each. After these images had been acquired, the thyroid phantoms were divided into 2 pieces (right and left lobes). The radioactivity of each lobe was then measured using an automatic well counter, and their volumes were measured using a measuring cylinder.

\section{Parathyroid Imaging}

We simulated the following 3 acquisition protocols: a dual-phase ${ }^{99 \mathrm{~m} T c-s e s t a m i b i}$ protocol, a dual-tracer ${ }^{99 \mathrm{~m}} \mathrm{Tc}$-sestamibi/ ${ }^{99 \mathrm{~m}} \mathrm{Tc}-$ pertechnetate protocol, and a dual-tracer ${ }^{99 \mathrm{~m}} \mathrm{Tc}$-sestamibi/ ${ }^{123} \mathrm{I}$-iodine protocol.

The tracer dose of each phantom in these 3 protocols is shown in Table 1. ${ }^{99 \mathrm{~m}} \mathrm{Tc}$-pertechnetate was substituted for ${ }^{99 \mathrm{~m}} \mathrm{Tc}$-sestamibi in all our phantom studies since both tracers have the same photo energy of ${ }^{99 \mathrm{~m}} \mathrm{Tc}$. All scanning procedures were performed using the CZT camera for $10 \mathrm{~min}$.

Dual-Phase ${ }^{99 m}$ Tc-Sestamibi Protocol. ${ }^{99 \mathrm{~m} T \mathrm{~T}-\text {-sestamibi accumu- }}$ lates in both parathyroid adenoma and thyroid tissue as a function of blood flow, gland size, and mitochondrial activity $(15,17)$. However,
${ }^{99 m}$ Tc-sestamibi washes out more rapidly from thyroid tissue than from parathyroid adenoma $(15,16)$. Because of the difference in washout rate from the 2 tissues, a parathyroid adenoma is detected more clearly in a delayed-phase image than in an early-phase image. Thus, we prepared 2 types of neck phantoms to mimic the early- and delayed-phase neck images.

We determined the radioactivity of the early-phase phantom on the basis of preliminary experiments, including prior thyroid imaging. The radioactivity of the thyroid gland in the delayedphase phantom was determined using a previous study for reference (25). In that study, the washout rate of ${ }^{99 \mathrm{~m}} \mathrm{Tc}$-sestamibi from thyroid tissue was 0.013 at $180 \mathrm{~min}$ after administration. This value was approximately $13 \%$ of the radioactivity of the earlyphase image (at $20 \mathrm{~min}$ after administration). Generally, no biologic washout of ${ }^{99 \mathrm{~m}} \mathrm{Tc}$-sestamibi was observed from the parathyroid adenoma; hence, we calculated the radioactivity of the parathyroid adenoma in the delayed phase on the basis of only physical decay of ${ }^{99 \mathrm{~m}} \mathrm{Tc}$, which was similar to the background. After calibration, these early and delayed neck phantoms were imaged.

Dual-Tracer ${ }^{99 m} T c$-Sestamibi ${ }^{99 m} T c$-Pertechnetate Protocol. Although ${ }^{99 \mathrm{~m}} \mathrm{Tc}$-sestamibi accumulates in both parathyroid adenomas and thyroid tissue, ${ }^{99 \mathrm{~m}} \mathrm{Tc}$-pertechnetate accumulates only in thyroid tissue, not in a parathyroid adenoma. Thus, by subtracting a ${ }^{99 \mathrm{~m}} \mathrm{Tc}-$ pertechnetate image from a ${ }^{99 \mathrm{~m}} \mathrm{Tc}$-sestamibi image, we could obtain an image of a parathyroid adenoma $(16,18)$.

We constructed 2 types of neck phantoms. A single-tracer phantom to imitate a ${ }^{99 \mathrm{~m}} \mathrm{Tc}$-pertechnetate image contained ${ }^{99 \mathrm{~m}} \mathrm{Tc}$ pertechnetate in the thyroid gland but not in the parathyroid adenoma. A dual-tracer phantom that simulated ${ }^{99 \mathrm{~m} T c-s e s t a m i b i}$ and ${ }^{99 \mathrm{~m}} \mathrm{Tc}$-pertechnetate images contained ${ }^{99 \mathrm{~m}} \mathrm{Tc}$-pertechnetate in both the thyroid gland and the parathyroid adenoma. We set reference markers on the neck phantom and scan bed to reproduce the position of phantoms consistently. First the single-tracer phantom was scanned. We then changed the single-tracer phantom to a dual-tracer phantom using the reference markers as a guide to

TABLE 2

Energy Windows Used with CZT and Nal Cameras

\begin{tabular}{|c|c|c|c|}
\hline \multirow[b]{2}{*}{ Window } & \multicolumn{2}{|c|}{ Single-tracer acquisition } & \multirow[b]{2}{*}{ Dual-tracer acquisition (CZT [keV]) } \\
\hline & CZT (keV) & Nal (keV) & \\
\hline $99 \mathrm{mTc}$ & $140.5 \% \pm 10 \%$ & $140.5 \% \pm 15 \%$ & $140.5 \% \pm 5 \%$ \\
\hline $123 \mid$ & $159 \% \pm 10 \%$ & $159 \% \pm 15 \%$ & $159 \% \pm 5 \%$ \\
\hline
\end{tabular}




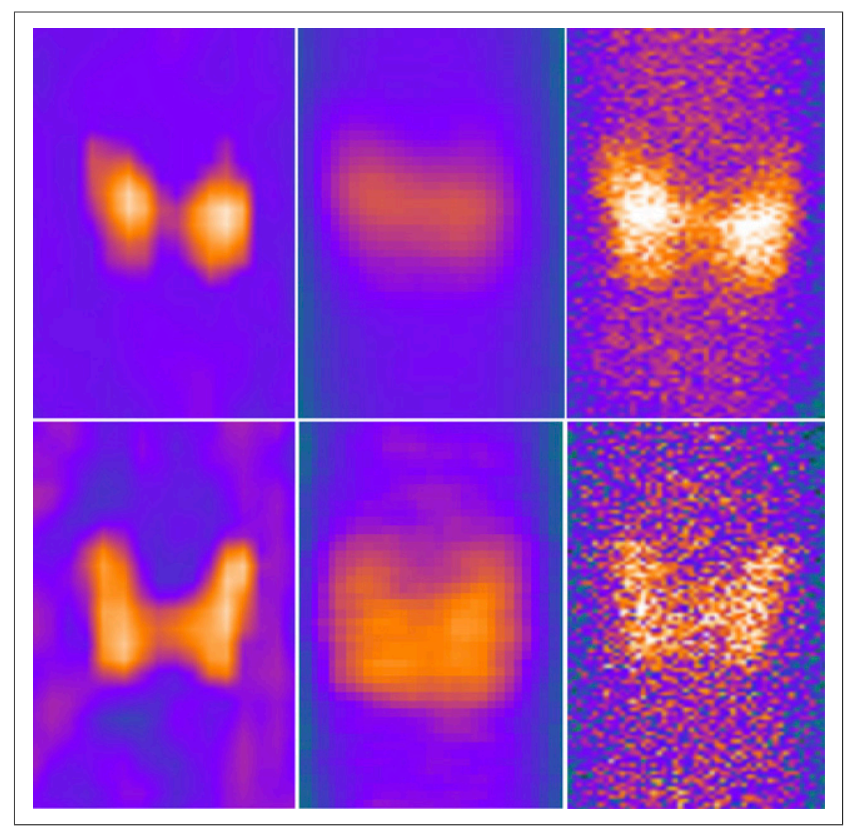
(bottom) tracers. Shown are MIP images (left), PE images (middle), and Nal planar images (right).

maintain their scan positions. The dual-tracer phantom was then imaged.

Dual-Tracer ${ }^{99 m}$ Tc-Sestamibi/123 I-Iodine Protocol. Sodium ${ }^{123}$ I-iodide accumulates only in thyroid tissue, and ${ }^{99 \mathrm{~m} T c-s e s t a m i b i}$ accumulates in both parathyroid adenoma and thyroid tissue. By subtracting a ${ }^{123}$ I-iodine image from a ${ }^{99} \mathrm{~m} \mathrm{Tc}$-sestamibi image, one can isolate the uptake in the parathyroid adenoma (16).

We prepared the thyroid phantom to include both ${ }^{99 \mathrm{~m}} \mathrm{Tc}-$ pertechnetate and ${ }^{123}$ I-BMIPP, whereas the parathyroid adenoma phantom included only ${ }^{99 \mathrm{~m}} \mathrm{Tc}$-pertechnetate. The background included both ${ }^{99} \mathrm{~m}$ Tc-pertechnetate and ${ }^{123} \mathrm{I}$-BMIPP. Dual-energy simultaneous acquisition of ${ }^{99} \mathrm{~m} \mathrm{Tc}$ and ${ }^{123} \mathrm{I}$ was performed. After acquisition, we generated ${ }^{99 \mathrm{~m}} \mathrm{Tc}$ and ${ }^{123} \mathrm{I}$ window images separately, and subtracted the ${ }^{123} \mathrm{I}$ window image from the ${ }^{99 \mathrm{~m}} \mathrm{Tc}$ window image.

\section{Image Acquisition and Processing}

In this study, we used D-SPECT (Spectrum Dynamics Medical) for CZT SPECT imaging. D-SPECT is equipped with tungsten parallel-hole collimators and uses list-mode acquisition, with 120 projections per detector. The matrix size of CZT SPECT images was $16 \times 64$, and the pixel size was $2.26 \mathrm{~mm} /$ pixel. CZT SPECT
FIGURE 1. Typical thyroid images using 99mTc (top) and ${ }^{123}$

data were reconstructed using ordered-subset expectation maximization, with 7 iterations and 32 subsets. When we performed dualtracer acquisition with the ${ }^{99 \mathrm{~m}} \mathrm{Tc}$ and ${ }^{123} \mathrm{I}$ windows, 2 different energy windows were used simultaneously without scatter correction. The NaI camera (BrightView; Philips) was equipped with a low-energy, high-resolution collimator. The matrix size of the planar image from the $\mathrm{NaI}$ camera (NaI planar image) was $256 \times 256$, and the pixel size was $6.4 \mathrm{~mm} /$ pixel. The details of the energy windows used for the CZT and NaI cameras are shown in Table 2.

All images were analyzed using freely downloadable software for nuclear medicine (Prominence Processor, version 3.1; Japanese Society of Radiologic Technology) (26). Using this software, we made maximum-intensity-projection (MIP) images from the CZT SPECT data by the same method as that used to make MIP images from the $\mathrm{NaI}$ data (27). The maximum intensity along the viewing path was projected. We also used the "planar H/M" application program, which was installed on the D-SPECT workstation (3). This application was programmed to evaluate the heart-to-mediastinum ratio in cardiac ${ }^{123}$ I-metaiodobenzylguanidine scintigraphy. Each detector of D-SPECT provides a small 2-dimensional (2D) image per angular position. Taken together, all the small 2D images that share the same angle yield wide 2D planar-equivalent (PE) images, which look similar to planar images from a $\mathrm{NaI}$ camera.

In thyroid imaging, we set a region of interest on both lobes of the thyroid gland and on the background and measured the mean count per pixel. Image count for the region of interest was calculated by subtracting the background mean count from the region-ofinterest mean count. In parathyroid imaging, image subtraction was performed using dual-tracer protocols on the MIP image. We adjusted the phantom position and uptake intensity between different photopeak images for subtraction using the software, and subjectively satisfactory images for diagnosis were thus obtained.

\section{Statistical Analysis}

The data were analyzed using a website for statistical computation (VassarStats; http://vassarstats.net/). Correlations between the radioactivity and image counts were assessed using the Pearson correlation coefficient $(r)$. Differences in correlation coefficients between images were assessed using the Fisher transformation test. A 2-tailed $P$ value of less than 0.05 was considered statistically significant.

\section{RESULTS}

\section{Thyroid Imaging}

Typical images obtained using 3 different methods are shown in Figure 1. MIP images could depict the thyroid gland with good image quality; however, PE images were inferior to MIP and NaI planar images in both the ${ }^{99 \mathrm{~m}} \mathrm{Tc}$ and the ${ }^{123} \mathrm{I}$ windows.

The relationship between radioactiv-
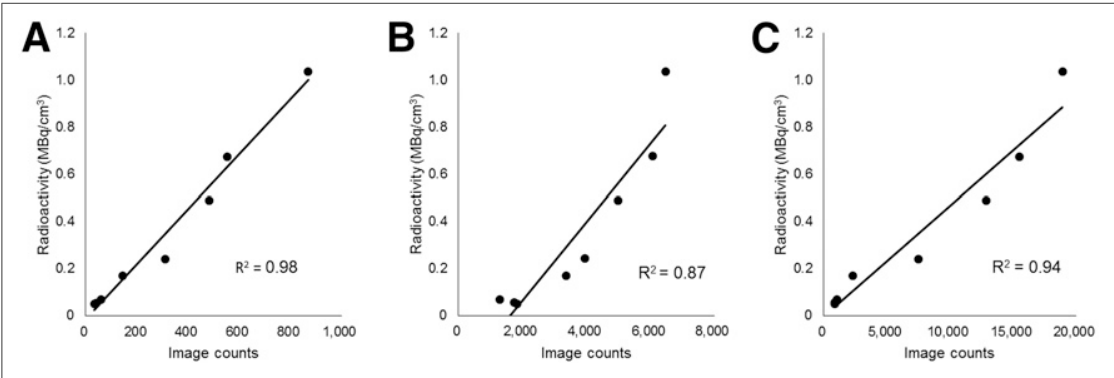

FIGURE 2. Relationship between image counts and radioactivity of ${ }^{99 m}$ Tc-containing thyroid phantoms: Nal planar image (A), MIP image (B), and PE image (C). ity and image counts is shown in Figures 2 and 3. MIP images and PE images showed statistically significant correlations between the radioactivity and image counts for both ${ }^{99 \mathrm{~m} \mathrm{Tc}}$ ( $r=0.93$ and 0.97 , respectively; $P<0.05)$ and ${ }^{123} \mathrm{I}$ images ( $r=0.89$ and 0.99 , respectively; $P<0.05)$. Similar results were obtained using the $\mathrm{NaI}$ planar images for the

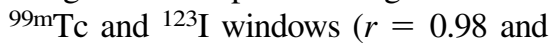




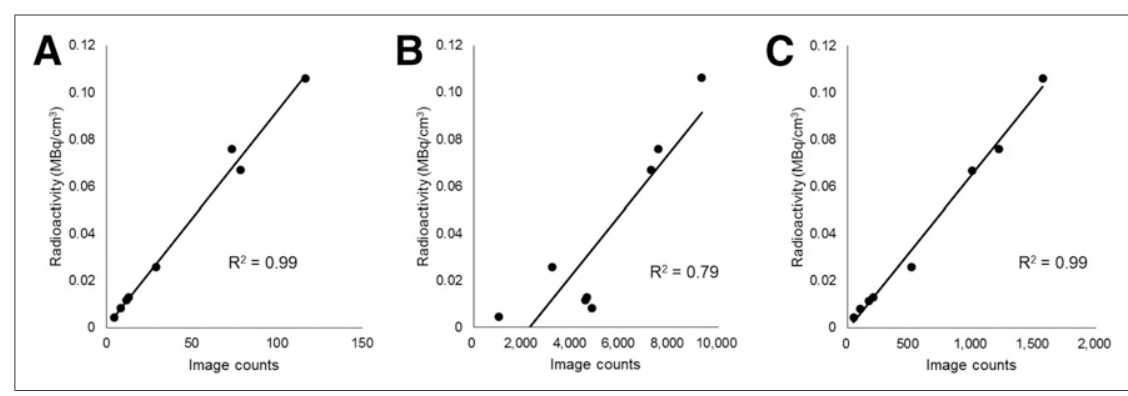

FIGURE 3. Relationship between image counts and radioactivity of 123 thyroid phantoms: Nal planar image (A), MIP image (B), and PE image (C). adenoma was difficult. After subtraction of the ${ }^{123}$ I photopeak image from the ${ }^{99 \mathrm{~m}} \mathrm{Tc}$ photopeak image, the parathyroid adenoma was discernable.

\section{DISCUSSION}

\section{Thyroid Imaging and \\ Quantitative Analysis}

Cardiac CZT SPECT does not directly generate planar images, and most thyroid imaging is performed using a $2 \mathrm{D}$ approach. Thus, we needed to convert tomographic data to $2 \mathrm{D}$ data 0.99 , respectively; $P<0.05$ ). The correlations between radioactivity and image counts of ${ }^{99 \mathrm{~m}} \mathrm{Tc}$ tracer did not significantly differ between MIP and PE images $(P=0.54)$. However, with the ${ }^{123} \mathrm{I}$ tracer, PE images had significantly stronger correlations than the MIP images $(P<0.05)$.

\section{Parathyroid Imaging}

Figure 4 shows the dual-phase ${ }^{99 \mathrm{~m}} \mathrm{Tc}$-sestamibi protocol. The early-phase image depicted tracer uptake in the thyroid gland, with regional emphasis on the parathyroid adenoma in the lower quadrant of the left thyroid lobe. A clear accumulation in the parathyroid adenoma was visible in the simulated delayed-phase image.

Figure 5 shows the dual-tracer ${ }^{99 \mathrm{~m}} \mathrm{Tc}$-sestamibi/ $99 \mathrm{~m} \mathrm{Tc}-$ pertechnetate protocol. Dual-tracer images showed tracer uptake in the thyroid gland and parathyroid adenoma, whereas single-tracer images showed tracer uptake in the thyroid gland only. Subtraction of the single-tracer image from the dual-tracer image showed the parathyroid adenoma unmistakably.

Figure 6 shows the dual-tracer ${ }^{99 \mathrm{~m} T c-s e s t a m i b i /}{ }^{123} \mathrm{I}-$ iodine protocol. The ${ }^{123}$ I photopeak image showed tracer uptake in the thyroid gland. In contrast, the ${ }^{99 \mathrm{~m}} \mathrm{Tc}$ photopeak image showed tracer uptake in the thyroid gland and parathyroid adenoma; however, identifying the parathyroid

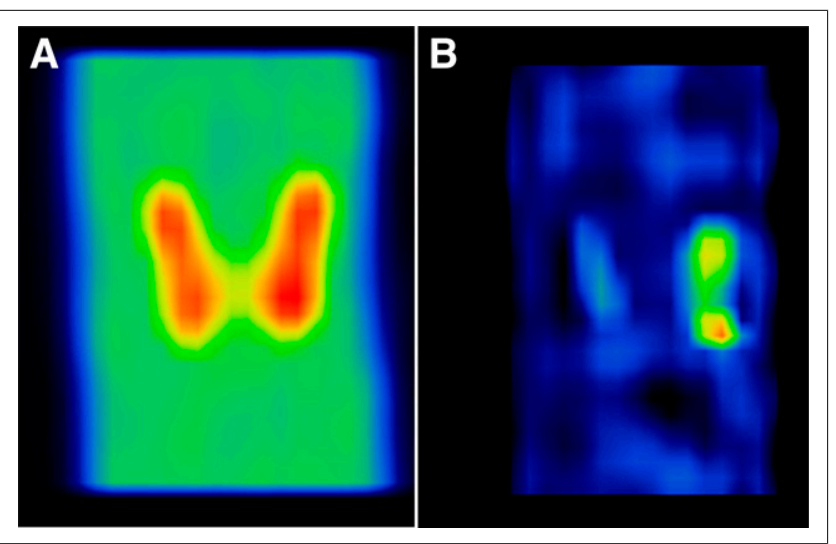

FIGURE 4. Typical early $(A)$ and delayed $(B)$ images from dualphase ${ }^{99 m}$ Tc-sestamibi protocol using MIP. Clear accumulation of tracer is visible in lower quadrant of left thyroid lobe in delayed image. for a corresponding thyroid assessment. After an MIP image was generated, it could depict the thyroid gland as effectively as images from a NaI camera. In contrast, PE images were not adequate for visual assessment of the thyroid gland. The reason for this phenomenon is unclear, because this technique is theoretically similar to that performed with a $\mathrm{NaI}$ camera (3). At the least it can be concluded that an MIP image needs to be generated for visual assessment of the thyroid gland using cardiac CZT SPECT.

Image counts measured by setting a region of interest on the CZT SPECT and NaI scans were significantly correlated in both the ${ }^{99 \mathrm{~m}} \mathrm{Tc}$ and the ${ }^{123} \mathrm{I}$ images. However, a detailed inspection revealed that in the MIP images, the regression line of the correlation did not cross the origin of the coordinates. In an MIP-image-visualized plane, because the voxels with maximum intensity that fall in the way of parallel rays trace from the viewpoint to the plane of projection, the count data might be overestimated. Thus, the approximate lines of the MIP image shifted to the higher-count area. In addition, the PE images produced stronger correlations of radioactivity with image counts for the ${ }^{123} \mathrm{I}$ tracer than for the ${ }^{99 \mathrm{~m}} \mathrm{Tc}$ tracer. PE images were developed especially for calculation of the mean pixel counts of ${ }^{123}$ I tracer (3). This property may be the reason for better quantification for the ${ }^{123}$ I tracer than

In this study, we obtained clear parathyroid adenoma images in our phantom experiments that faithfully reproduced 3 different acquisition protocols using a CZT camera (16).

With the dual-phase ${ }^{99 \mathrm{~m}} \mathrm{Tc}$-sestamibi protocol, a parathyroid adenoma can be visualized in a delayed-phase image. Because a dual-phase ${ }^{99 \mathrm{~m}} \mathrm{Tc}$-sestamibi protocol is the most popular imaging protocol for parathyroid adenomas, our result indicates that this protocol may be one of the most feasible methods for the imaging of other organs using a CZT camera instead of an NaI camera. To clarify this issue, further studies that include various sizes and radioactivities of a parathyroid adenoma are needed.

In the dual-tracer ${ }^{99} \mathrm{~m} \mathrm{Tc}$-sestamibi/99m $\mathrm{Tc}$-pertechnetate protocol, we also obtained a good image of a parathyroid adenoma. Although several position gaps appeared between for the ${ }^{99 \mathrm{~m}} \mathrm{Tc}$ tracer.

\section{Parathyroid Imaging}




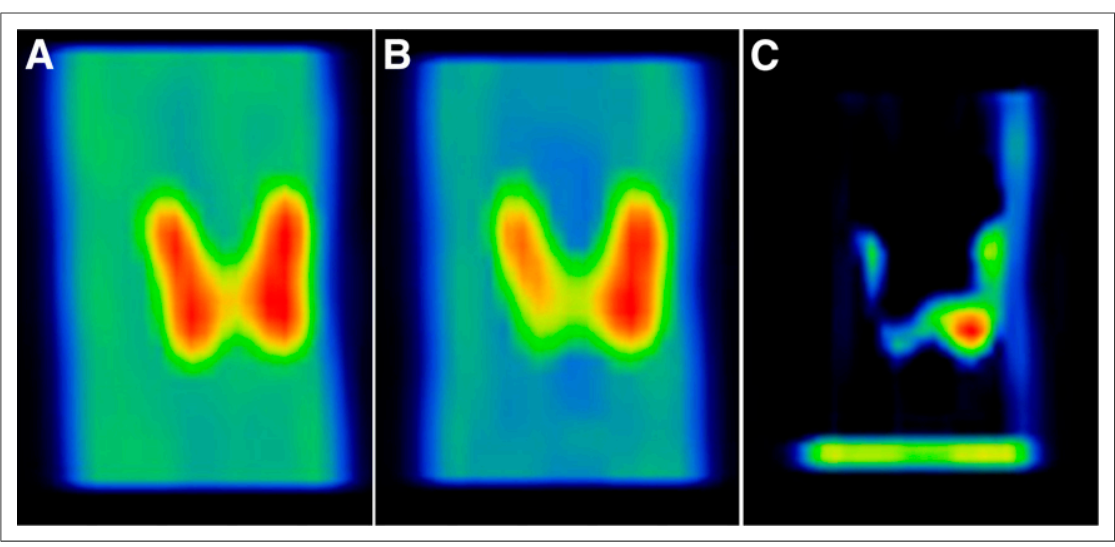

FIGURE 5. Typical single-tracer (A), dual-tracer (B), and subtracted (C) images from 99mTc-sestamibi/99mTc-pertechnetate protocol using MIP. After single-tracer image was subtracted from dual-tracer image, solitary parathyroid adenoma was clearly visible.

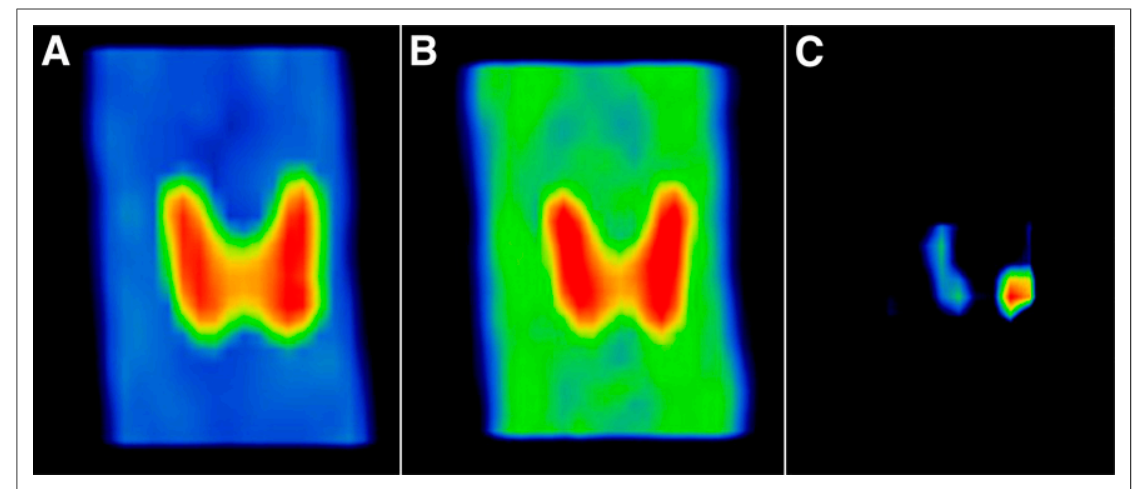

FIGURE 6. Representative ${ }^{99 m T c}$ photopeak (A), ${ }^{123}$ photopeak (B), and subtracted (C) parathyroid images from ${ }^{99 \mathrm{~m}} \mathrm{Tc}$-sestamibi/ ${ }^{123}$ /-iodine protocol using MIP. ${ }^{99 \mathrm{~m} T c}$ tracer is seen in thyroid and parathyroid glands, and ${ }^{123}$ is distributed in thyroid tissue. Parathyroid

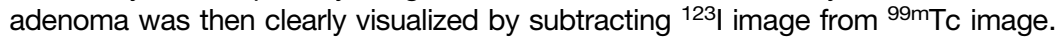

2 different neck phantoms, software-based registration can permit high-quality subtraction to clearly emphasize a parathyroid adenoma. Thus, this protocol also seems to be acceptable for parathyroid imaging by CZT SPECT. In the clinical setting, these 2 images are obtained without changing a patient's neck position, unlike in our study; thus, a much clearer and motionless parathyroid image would be expected in human imaging.

Because both images were acquired simultaneously, in the dual-tracer ${ }^{99 \mathrm{~m}} \mathrm{Tc}$-sestamibi/ ${ }^{123} \mathrm{I}$-iodine protocol a parathyroid adenoma was clearly visualized by subtracting the ${ }^{123} \mathrm{I}$ photopeak image from the ${ }^{99 \mathrm{~m}} \mathrm{Tc}$ photopeak image. Although the emission energies of these tracers are close and crosstalk has an influence on image quality, currently this protocol is often performed in routine practice using a NaI camera. Energy resolution is better for a CZT camera than for a NaI camera; thus, CZT SPECT is theoretically more suitable for the dualtracer protocol using ${ }^{99 \mathrm{~m}} \mathrm{Tc}$ and ${ }^{123} \mathrm{I}$. Tunninen et al. reported that the ${ }^{99 \mathrm{~m}} \mathrm{Tc}$-sestamibi/ ${ }^{123}$ I-iodine dual-tracer protocol is superior to the ${ }^{99 \mathrm{~m}} \mathrm{Tc}$-sestamibi single-tracer protocol (14). Taieb et al. also reported a high sensitivity using ${ }^{99 \mathrm{~m}} \mathrm{Tc}$-sestamibi/123 $/$-iodine subtraction scintigraphy (28). Therefore, the dual-tracer $99 \mathrm{~m} \mathrm{Tc}$-sestamibi//23I-iodine acquisition using CZT SPECT seems to be the best protocol for nuclear medicine imaging of the parathyroid. Further clinical studies are needed to clarify the clinical impact of this technology.

\section{Limitations}

In this study, we used the same tracer dose and acquisition time for both the CZT and the NaI cameras because this preliminary study needed to compare these 2 modalities under the same conditions. Theoretically, a protocol with a lower dose or shorter acquisition time can be used with CZT SPECT. Therefore, in future studies the tracer dose and acquisition time should be reduced to take advantage of CZT SPECT. Because the dual-tracer protocol increases the radiation exposure, we should attempt to reduce this with CZT SPECT technology.

It is well known that a certain proportion of patients with hyperparathyroidism have an ectopic parathyroid adenoma (29). If an ectopic parathyroid adenoma were in the mediastinum, CZT SPECT with a limited field of view would not cover both the neck and the mediastinum using one-time scanning. Typically, CZT SPECT is used for cardiac imaging; thus, it would be acceptable to perform additional mediastinal imaging as is done in a cardiac study. However, further research is required.

\section{CONCLUSION}

To our knowledge, this is the first study that attempted imaging of the neck region using cardiac CZT SPECT. A combination of MIP and PE images appears to be useful for visual and quantitative assessment of thyroid disease. MIP images can be applied for the detection of parathyroid adenomas using several different protocols, including the dual-tracer method.

\section{DISCLOSURE}

No potential conflict of interest relevant to this article was reported.

\section{ACKNOWLEDGMENT}

We thank Shoji Nono of the Department of Radiology, National Cerebral and Cardiovascular Center, for his technical assistance. 


\section{REFERENCES}

1. Bocher M, Brevis IM, Tsukerman L, Shrem Y, Kovalski G, Volokh L. A fast cardiac gamma camera with dynamic SPECT capabilities: design, system validation and future potential. Eur J Nucl Med Mol Imaging. 2010;37:1887-1902.

2. Koulikov V, Lerman H, Kesler M, Spair EE. ${ }^{99 \mathrm{~m} T c-M D P}$ bone scintigraphy of the hand: comparing the use of novel cadmium zinc telluride (CZT) and routine $\mathrm{NaI}(\mathrm{Tl})$ detectors. EJNMMI Res. 2015;5:63.

3. Bellevre D, Manrique A, Lagallois D, et al. First determination of the heart-tomediastinum ratio using cardiac dual isotope ( ${ }^{123} \mathrm{I}-\mathrm{MIBG} /{ }^{99 \mathrm{~m}} \mathrm{Tc}$-tetrofosmin) CZT imaging in patients with heart failure: the ADRECARD study. Eur J Nucl Med Mol Imaging. 2015;42:1912-1919.

4. Takahashi Y, Miyagawa M, Nishiyama Y, Kawaguchi N, Ishimura H, Mochizuki T. Dual radioisotopes simultaneous SPECT of ${ }^{99 \mathrm{~m}} \mathrm{Tc}$-tetrofosmin and ${ }^{123}$ I-BMIPP using a semiconductor detector. Asia Ocean J Nucl Med Biol. 2015;3:43-49.

5. Einstein AJ, Blankstein R, Andrews $\mathrm{H}$, et al. Comparison of image quality, myocardial perfusion, and left ventricular function between standard imaging and single-injection ultra-low-dose imaging using a high-efficiency SPECT camera: the MILLISIEVELT study. J Nucl Med. 2014;55:1430-1437.

6. Gambhir SS, Berman DS, Ziffer J, et al. A novel high-sensitivity rapid-acquisition single-photon cardiac imaging camera. J Nucl Med. 2009;50:635-643.

7. van Dijk JD, Jager PL, Ottervanger JP, et al. Minimizing patient-specific tracer dose in myocardial perfusion imaging using CZT SPECT. J Nucl Med Technol. 2015;43:36-40.

8. Kobayashi M, Matsunari I, Nishi K, et al. Simultaneous acquisition of ${ }^{99 \mathrm{~m} T c-}$ and ${ }^{123} \mathrm{I}$ - labeled radiotracers using a preclinical SPECT scanner with CZT detectors. Ann Nucl Med. 2016;30:263-271.

9. Ko T, Utanohara Y, Suzuki Y, et al. A preliminary feasibility study of simultaneous dual-isotope imaging with a solid-state dedicated cardiac camera for evaluating myocardial perfusion and fatty acid metabolism. Heart Vessels. 2016;31:38-45.

10. Tsai CJ, Cheng CY, Wang LY, et al. Tc-99m imaging in thyroidectomized differentiated thyroid cancer patients immediately before I-131 treatment. Nucl Med Commun. 2016;37:182-187.

11. Gerard SK, Cavalieri RR. ${ }^{123}$ I diagnostic thyroid tumor whole-body scanning with imaging at 6, 24, and 48 hours. Clin Nucl Med. 2002;27:1-8.

12. Bravo PE, Goudarzi B, Rana U, et al. Clinical significance of discordant findings between pre-therapy ${ }^{123} \mathrm{I}$ and post-therapy ${ }^{131} \mathrm{I}$ whole body scan in patients with thyroid cancer. Int J Clin Exp Med. 2013;6:320-333.

13. Ozdemir D, Cuhaci FN, Ozdemir E, et al. The role of postoperative ${ }^{99 \mathrm{~m}} \mathrm{Tc}$ pertechnetate scintigraphy in estimation of remnant mass and prediction of successful ablation in patients with differentiated thyroid cancer. Nucl Med Commun. 2016;37:640-645.
14. Tunninen V, Varjo P, Schildt J, et al. Comparison of five parathyroid scintigraphic protocols. Int J Mol Imaging. 2013;2013:921260.

15. Mariani G, Gulec SA, Rubello D, et al. Preoperative localization and radioguided parathyroid surgery. J Nucl Med. 2003;44:1443-1458.

16. Greenspan BS, Dillehay G, Intenzo C, et al. SNM practice guideline for parathyroid scintigraphy 4.0. J Nucl Med Technol. 2012;40:111-118.

17. Elgazzar AH, Anim JT, Dannoon SF, Farghaly MM. Ultrastructure of hyperfunctioning parathyroid glands: does it explain various patterns of ${ }^{99 \mathrm{~m}} \mathrm{Tc}$-sestamibi uptake. World J Nucl Med. 2017;16:145-149.

18. Keane DF, Roberts G, Smith R, et al. Planar parathyroid localization scintigraphy: a comparison of subtraction and 1-, 2-, and 3-h washout protocols. Nucl Med Commun. 2013;34:582-589.

19. Chaudhary V, Bano S. Thyroid ultrasound. Indian J Endocrinol Metab. 2013;17:219-227.

20. Vurdem UE, Acer N, Ertekin T, Savranlar A, Topuz O, Keceli M. Comparison of three volumetric techniques for estimating thyroid gland volume. Turk J Med Sci. 2012;42(suppl 1):1299-1306.

21. Spencer RP, Waldman R. Size and positional relationships between thyroid lobes in the adult as determined by scintillation scanning. J Nucl Med. 1965;6:53-58.

22. Fang L, Tang B, Hou D, Meng M, Xiong M, Yang J. Relationship between parathyroid mass and parathyroid hormone level in hemodialysis patients with secondary hyperparathyroidism. BMC Nephrol. 2015;16:82.

23. Randhawa PS, Mace AD, Nouraei SA, Sterns MP. Primary hyperparathyroidism: do perioperative biochemical variables correlate with parathyroid adenoma weight or volume? Clin Otolaryngol. 2007;32:179-184.

24. Fujii H, Iwasaki R, Ogawa K, et al. Evaluation of parathyroid imaging methods with Tc-99m-MIBI: the comparison of planar images obtained using a pinhole collimator and a parallel-hole collimator [in Japanese]. Kaku Igaku. 1999;36:425433.

25. Santos AO, Wittmann DE, Nogueria RO, et al. ${ }^{99 \mathrm{~m}} \mathrm{Tc}$ sestamibi thyroid uptake in euthyroid individuals and in patients with autoimmune thyroid disease. Eur $J$ Nucl Med Mol Imaging. 2005;32:702-707.

26. Maeda H, Yamaki N, Azuma M. Development of the software package of the nuclear medicine data processor for education and research [in Japanese]. Nihon Hoshasen Gijutsu Gakkai Zasshi. 2012;68:299-306.

27. Komori T, Narabayashi I, Doi K, Sueyoshi K, Tatsu Y, Utsunomiya K. A case of local recurrent pheochromocytoma: usefulness of I-123 MIBG early SPECT and maximum intensity projection images. Clin Nucl Med. 2000;25:285-287.

28. Taieb D, Hindle E, Grassetto G, Colletti PM, Rubello D. Parathyroid scintigraphy when, how, and why? A concise systematic review. Clin Nucl Med. 2012;37:568-574.

29. Grozavu C, Pantile D. Primary hyperparathyroidism through an ectopic parathyroid adenoma. Chirurgia (Bucur). 2016;111:156-160. 